\title{
The advantage of specific terms over general terms as cues for sentence recall: Instantiation or retrieval?
}

\author{
WILLIAM E. GUMENIK \\ University of Toledo, Toledo, Ohio 43606
}

\begin{abstract}
Anderson et al. (1976) found that specific terms, which had not appeared in but fit the context of sentences, were better sentence recall cues than the general terms appearing in the sentences. They interpreted this as indicating that general terms in context were encoded on the basis of appropriate instantiations. Experiment 1, using part of the same material, demonstrated that the advantage of specific cues increased when the general terms were omitted from the to-be-remembered materials, and that other specific terms were also effective cues for the same material. Experiment 2 demonstrated that terms that could not be possible intantiations of any words in the sentences, but that shared common meaning with the sentences as a whole, were as effective retrieval cues as the specific terms. The advantage of specific cues was interpreted as attributable to feature overlap between the specific terms and the to-beremembered materials rather than to instantiation of the general terms.
\end{abstract}

Anderson, Pichert, Goetz, Schallert, Stevens, and Trollip (1976) held that words have whole families of related meanings rather than fixed meanings. In the case of nouns, the context often limits the meaning of the noun to a particular subset of potential meanings, through inferences from "schemata" encompassing one's knowledge of language and the world. "If the context is rich and the message is processed deeply, a noun may be identified with a single real or imagined thing" (Anderson et al., 1976, p. 667). This process is called "instantiation" and is held by Anderson et al. to be integral to sentence comprehension and memory.

Anderson et al. (1976) ran a series of experiments aimed at determining whether a general term presented in context is actually encoded and stored in terms of one of its exemplars. As recall cues, they pitted an exemplar that fit the context of the sentence but was not presented at input against the actually presented general noun that had served as the sentence subject. For example, the relative efficacies of the cues "shark" and "fish" for the sentence, "The fish attacked the swimmer," were compared. They reasoned that if an appropriate instance of the category denoted by the sentence subject was encoded in the place of the actually presented general noun, this exemplar would be a better recall cue than the general noun. Cued recall was found

The author wishes to thank Laurie Crider, Elizabeth Fitkin, Mary Houghtell, Lynnette Johnson, Linda Maxon, Carol McGinn, Mark Miller, Alan Monday, Anda Rebensal, Helen Russell, Frank Wester, and Debra Zielinsky for their assistance in data collection, data tabulation, and/or judging the correctness of responses for Experiment 1 . The author also wishes to thank Daniel Nordlund for similar assistance in Experiment 2. Reprint requests should be sent to William E. Gumenik, Department of Psychology, University of Toledo, Toledo, Ohio 43606. to be significantly better using the exemplars rather than the actually presented general nouns, thus supporting an instantiation explanation. In line with this explanation, they also found that exemplars that fit the sentence context made better cues than those that did not.

In contrast to the cued recall data, Anderson et al. (1976) found that in free recall of the entire sentences, the actually presented general noun was produced rather than the hypothesized exemplar in all but $11 \%$ of the cases in which the sentences were recalled. In order to account for this, they postulated a persistent trace for the surface form of the message. This explanation entails the storage of the sentence subject in two different fashions simultaneously: in terms of the actually presented noun on a surface level, and its most appropriate exemplar on a deeper level. Since the free recall data clearly demonstrated that the actually presented general noun was accessible, it is difficult to understand why it was a poorer cue than the exemplar.

There has been much support for the theoretical position that there is storage of the meaning of a message, aside from its wording (e.g., Bransford, Barclay, \& Franks, 1972; Bransford \& Franks, 1971; Kintsch, 1974; Sachs, 1967). On the basis of this, one could provide an alternate explanation of the results of Anderson et al. (1976) in terms of a retrieval phenomenon, without postulating any encoding and storage of a nonpresented exemplar at input. According to this explanation, an exemplar that fits the context of the sentence would be a better recall cue than the actually presented general noun, because its meaning has more in common with the meaning of the entire presented message. For example, for the sentence, "The fish attacked the swimmer," the term "shark," which is a fish that is known to be aggressive and to attack 
swimmers, would have more features in common with the sentence than the actual sentence subject, "fish." Therefore, "shark" would be a more effective cue. The same reasoning would apply to the case of an exemplar that fits the context of the sentence being a better cue than one that does not.

Anderson et al. (1976) recognized that their results might be attributable to a retrieval phenomenon rather than instantiation of the general term at encoding. In order to eliminate the possibility that the advantage of the instantiation cue was attributable to preexisting associations between that cue and any of the words constituting the sentence, they included two kinds of control sentences. In one, the only word that was changed from the initial sentence was the verb, and in the other, the only word, aside from articles, that was retained was the verb. For example, for the sentence, "The fish attacked the swimmer," the two control sentences were "The fish avoided the swimmer" and "The communists attacked the village." While these control sentences rule out the possibility that the results were a retrieval phenomenon attributable to preexperimental associations between the instantiation cue and any word in the sentence, the control sentences also greatly changed the meaning of the test sentence. Therefore, the control sentences did not eliminate the possibility that the results were a retrieval phenomenon attributable to greater commonality of meaning between the entire sentence and the word selected as the instantiation cue. It is apparent that such commonality of meaning between the selected exemplar and the sentence context is inherent in the procedure of attempting to use the sentence context to guide a specific instantiation.

Differential predictions can be made between the presently proposed retrieval explanation and the instantiation explanation of Anderson et al. (1976). The retrieval explanation would hold that appropriate exemplars should remain better recall cues than general nouns, as long as the exemplars share more features in common with the to-be-recalled materials. This should occur even if the to-be-recalled materials consist only of the predicates of the sentences and there is no opportunity to instantiate the sentence subjects. In other words, "shark" should still be a better cue than "fish" because it shares more features in common with the phrase, "attacked the swimmer," whether or not this phrase is preceded by the sentence subject, "fish." In fact, the difference in efficacy between the exemplars and general nouns should be accentuated, since any advantage accruing to the general noun from its contiguity to the predicate at input, when the entire sentence is presented, would be removed when only the predicate is presented.

Also, according to the instantiation explanation, the advantage of the exemplar over the actually presented general noun as a recall cue is attributable to the substitution of that particular exemplar for the general noun at input. A different, less accessible exemplar, therefore, would not be expected to be an effective cue. According to the retrieval explanation, any exemplars that had more features in common with the to-be-recalled materials than the general noun should be more effective cues. For example, both "shark" and "barracuda" would be expected to be better cues than "fish" for the to-be-recalled "fish attacked the swimmer" and "attacked the swimmer."

Experiment 1, using a portion of the sentences used by Anderson et al. (1976), examined cued recall under conditions in which presentation of the entire sentences vs. presentation of the predicates alone was manipulated factorially with three kinds of recall cues: the original sentence subject, the exemplars used by Anderson et al., and alternate exemplars that fit the context of the sentences.

\section{EXPERIMENT 1}

\section{Method}

Subjects. The subjects were 90 undergraduates at the University of Toledo. They participated in the experiment voluntarily, without receiving class credit or monetary renumeration. They were randomly assigned to six treatment groups of 15 subjects each.

Materials. The to-be-recalled materials were either 20 complete sentences or 20 corresponding phrases in which the initial article, "the," and the subject noun were omitted. Thirteen of the sentences corresponded to 13 of the Anderson et al. (1976, Experiment 2) target sentences, which had been constructed to encourage a particular instantiation of the subject noun. These 13 and the 13 corresponding phrases served as test items. The remaining items were filler items.

Test booklets consisted of 20 cues, 1 cue per page. The cues were either the sentence subjects themselves ( $G$ cues) or possible exemplars of the categories denoted by the sentence subjects. The exemplar cues were of two types, $P$ or $P^{\prime}$. In the case of the test items, the $P$ cues were the same as the "particular" cues used by Anderson et al. (1976), which had been selected by them as the most appropriate instantiations of the sentence subjects in the sentence contexts. The $\mathbf{P}^{\prime}$ cues were alternative exemplars selected by the author, which also could fit the context of the sentence. The cues for the filler items were of a similar nature. Each test booklet consisted of only one kind of retrieval cue $\left(G, P\right.$, or $\left.P^{\prime}\right)$. The to-be-recalled test materials and their corresponding cues are presented in Appendix A.

A filler task, which was presented between the acquisition and test phases of the experiment, consisted of 15 sequences of numbers, each with a logical pattern of development. The task required the production of the next number in the sentence.

Design and Procedure. There were six treatment groups corresponding to the factorial arrangement between two kinds of input conditions (sentence or phrase) by three types of recall cues $\left(G, P\right.$, or $\left.P^{\prime}\right)$. Subjects were tested in groups of three, six, or nine. All subjects tested simultaneously were tested under the same input condition but were equally divided among the three cue conditions. The input conditions were block randomized among groups.

The experiment was given under the guise of an investigation of the relationship between verbal memory processes and mathematical reasoning. The subjects were instructed to pay close attention to the input materials since they would be asked to 
recall them later. The sentences or phrases were read aloud to the subjects, with a $7-\mathrm{sec}$ interval between the beginning of each item. The items were always read in the same order.

A 5-min mathematical-reasoning filler task occurred between the acquisition and test conditions.

In the test conditions, the subjects received a test booklet consisting of the $G, P$, or $P^{\prime}$ cues. The order in which the retrieval cues were presented in the booklets varied among different subjects in each condition. Next to each cue, the subjects were to write the previously presented sentence or phrase of which it reminded them. They were allowed $10 \mathrm{~min}$ to complete the task.

The criterion used for determining correctness of response was whether the substance of the response was in agreement with the predicate of the sentence. Since the investigation was concerned with the retrieval of the meaning of the to-be-recalled material, a substance rather than verbatim criterion for accuracy of the response was felt to be appropriate. Responses that corresponded to presented materials but were paired with the wrong retrieval cue were considered incorrect. Usually, the decision of correct or incorrect was simple and clear-cut. All responses were judged by at least two judges.

\section{Results}

The mean number of correct responses for each type of recall cue in the sentence and phrase conditions is shown in Table 1.

The exemplars, $\mathrm{P}$ and $\mathrm{P}^{\prime}$ cues, resulted in better recall than the general $G$ cues, with the $P$ cues resulting in better recall than $\mathrm{P}^{\prime}$ cues. The differences among the cues were greater in the phrase than in the sentence condition.

A 3 by 2 analysis of variance on these data revealed that the type of cue and input condition main effects were significant at the .01 level $[F(2,84)=14.994$, and $\mathrm{F}(1,84)=12.775$, respectively]. The Cue by Input interaction was not significant at the .05 level $[F(2,84)=$ $1.615, \mathrm{MSe}=5.992] . \mathrm{A}$ quasi-F analysis (Clark, 1973) revealed similar findings. The cue effect was significant at the .01 level, the input effect was significant at the .05 level, and the interaction was not significant $\left[\min F^{\prime}(2,131)=8.291, \min F^{\prime}(1,131)=4.320\right.$, and $\min F^{\prime}(2,131)=.993$, respectively] .

In order to determine whether the present results replicated the results of Anderson et al. (1976) using the same materials, an a priori comparison was run between the $G$ and $P$ retrieval cues in the sentence condition. In agreement with Anderson et al., this effect was significant $[F(1,84)=6.431, p<.05]$.

\section{Discussion}

Using a portion of the sentences utilized by Anderson et al. (1976), the present experiment replicated their findings that $P$ cues (exemplars) were better recall cues than $G$ cues (the general nouns that actually served as the sentence subjects at input). However, even when the sentence subjects were omitted at input and the to-beremembered materials were phrases composed of the predicates of the sentences, the advantage of $P$ cues over $G$ cues remained at least as great. These results are consistent with the retrieval explanation. If the $\mathrm{P}$ cues
Table 1

Mean Number of Test Items Correct

\begin{tabular}{lrrr} 
& \multicolumn{3}{c}{ Recall Cues } \\
\cline { 2 - 4 } & $\mathrm{G}$ & $\mathrm{P}$ & $\mathbf{P}^{\prime}$ \\
\hline Sentences & 3.60 & 5.87 & 4.27 \\
Phrases & .67 & 5.20 & 2.33 \\
\hline
\end{tabular}

shared more meaning than did the $G$ cues with the predicates of the sentences, then $P$ cues should be better retrieval cues than $G$ cues, whether the entire sentence or only the predicates were the to-be-recalled material.

Since the general noun sentence subjects were not presented in the phrase condition, there was no opportunity for them to be instantiated. On the basis of the instantiation explanation forwarded by Anderson et al. (1976), the most likely prediction would therefore appear to be that the advantage of $P$ cues should disappear in the phrase condition.

Anderson et al. (1976) could still maintain an instantiation explanation of this result by holding that there was an implicit subject that was instantiated at the input in the phrase condition. In other words, the phrase "protruded from the corpse" could have been interpreted as "something protruded from the corpse," "something" then being instantiated as "knife." If this were the case, the implicit subject in the phrase condition should be at least as specific as the actual sentence subject in the sentence condition. Otherwise, the range of possible instantiations would be expanded, rendering it less likely that the instantiation selected by the experimental subject would coincide with the recall cue selected by the experimenter. The term "weapon" in the sentence, "The weapon protruded from the corpse," at least rules out exemplars such as "a swollen tongue."

Another finding of the present experiment was that the efficacy of alternate exemplars ( $\mathbf{P}^{\prime}$ cues) to those used by Anderson et al. (1976) as recall cues fell between those of $P$ and $G$ cues in both the sentence and phrase conditions. This also is in agreement with the retrieval explanation. According to the retrieval explanation, any exemplar that had more features in common than the general noun with the meaning of the to-be-recalled material would be expected to be a more effective cue than the general noun. Anderson et al. constructed their $P$ cues and sentences so that the $P$ cues would be the most appropriate exemplars for their sentences. Other exemplars of the general nouns serving as sentence subjects, including the exemplars selected as $P^{\prime}$ cues in the present experiment, would not be expected to be as appropriate to the sentence context. Therefore, according to the retrieval explanation, the efficacy of $\mathbf{P}^{\prime}$ cues would be expected to fall between that of $P$ cues and $G$ cues.

According to the instantiation hypothesis, in the appropriate context, a narrowed subset of items is 
substituted for a general term at input. It is not necessary that the context narrow the subset to a single thing or to homogeneous things. However, if it is maintained that the word corresponding to the thing represented by the $P$ cue is instantiated for the general noun at input, the $P$ cue should serve as a better recall cue than the general noun because of redintegration. In fact, this appears to be the mechanism implied by Anderson et al. (1976) to account for their results. This explanation, however, could not account for the efficacy of $\mathrm{P}^{\prime}$ cues.

If, alternatively, it is held that the instantiation process does not result in the substitution of a word corresponding to $\mathbf{P}$ cues for the general terms at input, the mechanism by which the $P$ cue emerges as a better recall cue than the $G$ cue becomes unclear. It is possible that the narrowed subset represented by a concept or word is substituted for the general term at input. Then the $\mathbf{P}$ and $\mathbf{P}^{\prime}$ cues, during retrieval, elicit this subset, which redintegrates the sentence. However, the problem remains of why this subset would be more easily elicited by the $P$ and $P^{\prime}$ cues than by the $G$ cue.

Experiment 2 was addressed to a further implication of the retrieval explanation. According to the retrieval explanation, any terms that have more features in common with the meaning of the entire to-be-remembered message than the actually presented words constituting the messages should be more effective retrieval cues. It should make little difference whether or not these terms are possible instantiations of any of the actual words constituting the message.

\section{EXPERIMENT 2}

In Experiment 2, a comparison was made between the retrieval efficacy of cues that could not possibly be instantiations of any words in the to-be-remembered sentences but shared meaning with the sentence as a whole (W cues) and the retrieval efficacy of cues corresponding to the actually presented sentence subjects ( $G$ cues) and their possible instantiations ( $P$ cues). For example, "racing" would be the $W$ cue for the sentence, "The animal won by three lengths." The $G$ and $P$ cues, respectively, would be "animal" and "horse." Both W and P cues were selected on the basis of sharing more meaning in common with the entire sentence than the actually presented sentence subjects did. Therefore, according to the retrieval explanation, it should make little difference whether a $\mathrm{W}$ or a $\mathrm{P}$ cue is used. Both should be more effective retrieval cues than the $\mathrm{G}$ cues.

\section{Method}

Subjects. The subjects were 127 undergraduates enrolled in two introductory psychology classes at the University of Toledo. Each class was tested en masse, with the subjects assigned to one of three treatment groups (W, P, or G) on the basis of seating arrangement (i.e., subjects three seats apart received the same treatment condition). Because of a minor error in the order of handing out the test booklets, there were only 41 subjects in the $P$ condition, while there were 43 subjects in each of the other conditions.

Procedure. The procedure was essentially the same as in Experiment 1, with the exception of the changes described below. The presentation materials were 20 sentences, 15 of which were test items. The remaining five sentences were filler items, three of these being presented in the first three presentation positions and two in the final two positions. Eleven of the 15 test sentences were new; 4 were test sentences that had been used in Experiment 1. There were three types of test booklets: W cues, $P$ cues, and $G$ cues. The test booklets contained only cues for the 15 test sentences and not for the five filler sentences. The to-be-remembered materials and the cues for Experiment 2 are included in Appendix B.

The responses were scored by two independent raters, again using a substance rather than verbatim criterion for correctness of response. Interrater reliability between the two judges was high $(\mathrm{r}=.98)$.

\section{Results}

The mean number correct for the $\mathrm{W}, \mathrm{P}$, and $\mathrm{G}$ cues, respectively, was $5.91,5.95$, and 3.67. An unweightedmeans analysis of variance revealed the treatment effect to be significant at the .01 level $[F(2,124)=9.582$, $\mathrm{MSe}=7.485]$. A quasi-F analysis was also run on the data, after the number of subjects in each treatment group was equalized. This was accomplished by eliminating the data of two subjects in each of the $G$ and $P$ conditions through the use of a table of random numbers. As a result, the mean correct was slightly reduced in both groups. With elimination of these data, the mean correct became 5.85 for the P group and 3.51 for the $G$ group. This analysis also showed a significant cue effect $\left[\min \mathrm{F}^{\prime}(2,61)=3.542, \mathrm{p}<.05\right]$.

\section{DISCUSSION}

Experiment 2 indicated that it was not necessary for a retrieval cue to be a possible instantiation of any term in a sentence in order to be a more effective retrieval cue than the general noun that served as sentence subject. In fact, it made little difference whether the retrieval cue coincided with a possible instantiation of an actually presented term or not, as long as the retrieval cue appeared to share more meaning in common with the entire message than did the actually presented sentence subject.

These results, along with the results of Experiment 1, create some problems for an instantiation explanation. At a minimum, further development of the instantiation explanation would be necessary to account for the present results. It does not appear parsimonious to attribute the greater efficacy of other retrieval cues than the actually presented noun to instantiation of the sentence subject under conditions in which: (1) the exemplar remains an effective retrieval cue when the sentence subject to be instantiated is omitted from the presentation of the to-be-remembered materials; 
(2) other exemplars of the sentence subjects than their most probable instantiations are almost equally as effective as retrieval cues; (3) retrieval cues that are not possible instantiations of any words comprising, but that share meaning with, the whole sentences are equally as effective as the most probable instantiations of the actual sentence subjects and are both more effective than the actual sentence subjects. On the other hand, these findings can all be simply explained as a retrieval phenomenon in which the efficacy of the retrieval cues is a function of their shared meaning with the entire message.

In the present experiment, the decision about shared meaning was made on an intuitive basis. However, examination of the stimulus materials should convince the reader that $W, P$, and $P^{\prime}$ cues have more meaning in common with the whole to-be-remembered message than do G cues (see Appendix A, Appendix B). Future research might be directed toward an analysis of retrieval cues and to-be-remembered material into their com- ponent features of meaning, followed by an examination of recall as a function of their common and distinctive features.

\section{REFERENCES}

Anderson, R. C., Pichert, J. W.. Goetz, E. T., Schallert, D. J.. Stevens, K. V..\& Trollip. S. R. Instantiation of general terms. Journal of Verbal Learning and Verbal Behavior, 1976, 15, 667-679.

Bransford, J. D., Barclay, J. R., \& Franks, J. J. Sentence memory: A constructive versus interpretive approach. Cognitive Psychology, 1972, 3, 193-209.

Bransford, J. D., \& Franks, J. J. The abstraction of linguistic ideas. Cognitive Psychology, 1971, 2, 331-350.

Clark, H. H. The language-as-tixed-effect fallacy: A critique of language statistics in psychological research. Journal of Verbal Leaming and Verbal Behavior, 1973, 12. 335-359.

$\mathrm{KINTSCH}, \mathrm{W}$. The representation of meaning in memory. Hillsdale: Erlbaum. 1974.

SACHS, J. D. S. Recognition memory for syntactic and semantic aspects of connected discourse. Perception \& Psychophysics, 1967, 2. 437-442.

(Accepted for publication February 9, 1979.)

Appendix A

Test Sentences and Retrieval Cues for Experiment 1

\begin{tabular}{|c|c|c|c|c|}
\hline \multirow[b]{2}{*}{ Sentences } & \multirow{2}{*}{$\begin{array}{l}\text { Ordinal } \\
\text { Position }\end{array}$} & \multirow{2}{*}{$\begin{array}{c}\text { Test Items } \\
\mathbf{P}\end{array}$} & \multicolumn{2}{|c|}{ Recall Cues } \\
\hline & & & $\mathbf{G}$ & $\mathbf{P}^{\prime}$ \\
\hline The man planned the house. & 4 & architect & $\operatorname{man}$ & builder \\
\hline The leaves were smoked by the dropout. & 5 & marijuana & leaves & tobacco \\
\hline The group built their houses out of ice. & 6 & eskimo & group & polar expedition \\
\hline The fish attacked the swimmer. & 7 & shark & fish & barracuda \\
\hline The weapon protruded from the corpse. & 9 & knife & weapon & spear \\
\hline The vegetable is an important ingredient in salads. & 10 & lettuce & vegetable & tomato \\
\hline The utensil is required for soup. & 11 & spoon & utensil & ladle \\
\hline The fruit was made into wine. & 12 & grapes & fruit & cherries \\
\hline The woman was outstanding in the theatre. & 13 & actress & woman & ballerina \\
\hline The substance cleaned the clothes. & 14 & detergent & substance & bleach \\
\hline The game involves protecting the goal. & 15 & hockey & game & soccer \\
\hline The animal shook hands with its paws. & 16 & $\operatorname{dog}$ & animal & trained bear \\
\hline The container held the apples. & 18 & basket & container & carton \\
\hline
\end{tabular}

Note-When the phrases served as stimuli, the initial article and noun were omitted from presentation. Ordinal position refers to the position of the sentence in the acquisition stage.

Appendix B

Test Sentences and Retrieval Cues for Experiment 2

\begin{tabular}{|c|c|c|c|c|}
\hline \multirow[b]{2}{*}{ Sentences } & \multirow{2}{*}{$\begin{array}{l}\text { Ordinal } \\
\text { Position }\end{array}$} & \multirow{2}{*}{$\begin{array}{c}\text { Test Items } \\
\mathrm{W} \\
\end{array}$} & \multicolumn{2}{|c|}{ Recall Cues } \\
\hline & & & G & $\mathbf{P}$ \\
\hline The leaves were smoked by the dropout. & 4 & stoned & leaves & marijuana \\
\hline The group built their houses out of ice. & 5 & arctic & group & eskimos \\
\hline The fish attacked the swimmer. & 6 & jaws & fish & shark \\
\hline The weapon was protruding from the corpse & 7 & murder & weapon & knife \\
\hline The professional defended the accused. & 8 & trial & professional & lawyer \\
\hline The country sent a rocket to Mars. & 9 & aerospace & country & United States \\
\hline The animal won by three lengths. & 10 & racing & animal & horse \\
\hline The vehicles had to obey a $55 \mathrm{mph}$ speed limit. & 11 & energy shortage & vehicles & cars \\
\hline The woman paid for the supplies at the checkout counter. & 12 & supermarket & woman & shopper \\
\hline The food was scrambled and served with bacon. & 13 & breakfast & food & eggs \\
\hline The elected official ordered the atomic missiles to be launched at Moscow. & 14 & war & elected official & President \\
\hline The charity provided food, shelter, and medical care for the survivors. & 15 & disaster & charity & Red Cross \\
\hline The piece had to be moved to avoid checkmate. & 16 & chess & piece & king \\
\hline The organization raised the price of their product. & 17 & inflation & organization & manufacturer \\
\hline The man spoke against the bill for hours. & 18 & fillibuster & $\operatorname{man}$ & senator \\
\hline
\end{tabular}

Note-Ordinal position refers to the position of the sentence in the acquisition stage. 